\title{
DECLINO E INNOVAZIONE POLITICA NEL PARTITO LABURISTA INGLESE
}

\author{
di Oreste Massari
}

La vicenda del Labour Party degli anni ottanta costituisce un caso altamente paradigmatico, al di là della peculiarità della sua forma organizzativa (partito confederato e indiretto) e del contesto politico-istituzionale in cui opera (Allum 1988), di molteplici problemi che la scienza politica ha sollevato negli ultimi decenni circa la prospettiva del partito di massa e di quello specificamente ad origine di classe. Le tesi del declino del partito di classe e dell'esaurirsi dell'opposizione politico-parlamentare (Massari 1990a) sembravano calzare perfettamente alla parabola sempre più declinante del Labour Party a partire dalla metà degli anni settanta fino a culminare nel quasi collasso dei primi anni ottanta. Nel 1981 il partito subisce una pesante scissione alla sua destra, con la formazione dell'SDP, che con i liberali formerà l'Alliance elettorale. Nelle elezioni del 1983 il partito rischiò seriamente di essere scavalcato dall'Alliance finendo al terzo posto e di perdere il ruolo di opposizione ufficiale. Il suo voto si restrinse, infatti, a solo 8 milioni e mezzo $(27,6 \%$, il più basso risultato della sua storia elettorale dal 1918) a fronte di 7 milioni e ottocentomila voti dati all'Alliance $(25,4 \%)$ e a fronte di una sostanziale stabilità del voto conservatore $(42,4 \%)$. Il 1983 sembrò, difatti, un punto di svolta sia riguardo al futuro del Labour sia riguardo alla natura del sistema partitico. Occorre guardare alla geografia elettorale per comprendere appieno la portata della trasformazione. Se a livello parlamentare il sistema bipartitico ancora reggeva - avendo ottenuto conservatori e laburisti assieme 606 dei 650 seggi, grazie agli effetti disproporzionali del sistema elettorale - ciò non era più vero al livello dei collegi. A questo livello, il sistema bipartitico sembrava definitivamente rotto. Occorre avere presente che nella competizione maggioritaria ad un turno l'interesse va rivolto non solo, ovviamente, al partito che è arrivato primo

RIVISTA ITALIANA DI SCIENZA POLITICA / a. XXIII, n. 1, aprile 1993 
alla conquista del seggio, ma anche a chi è arrivato secondo, perché è questo che presumibilmente avrà le chances dell'alternanza al secondo round elettorale. L'Alliance, pur avendo ottenuto solo 23 seggi - e da questo punto di vista il Parlamento formatosi nel 1983 è risultato il meno «proporzionale» del mondo democratico (McLean 1988) - è arrivata seconda in 312 collegi, ossia in più della metà del totale. Il Labour arrivò primo in 209, secondo in 132, ma terzo (o anche peggio) in 292 collegi, corrispondenti a quasi metà dei seggi della Gran Bretagna.

Nell'Inghilterra meridionale, esclusa l'area di Londra, ossia nella parte più popolosa e sviluppata del paese, il Labour ottenne solo due seggi su 153, arrivando secondo solo in 18 (Berrington 1983). Solo la concentrazione del voto in Scozia, Galles e nel nord dell'Inghilterra, dovuta sia al radicamento sociale tradizionale sia alla crescente opposizione antithatcheriana nelle zone più emarginate del paese, ha permesso al partito laburista di conquistare i suoi 209 seggi. Ma proprio tale concentrazione di voti, in quelle aree che nelle elezioni del 1987 diverranno ancora di più le sue roccaforti, ha rivelato che nel 1983 il Labour aveva cessato di essere un partito nazionale, configurandosi come un partito solo regionale, legato all'archeologia industriale e al tradizionalismo del vecchio unionismo. In effetti, dunque, il 1983 vide la rottura del tradizionale two-party system (già apparso eroso alla fine degli anni sessanta) e l'emergenza di due paralleli sistemi bipartitici: in metà del paese, nella parte industriale, quello imperniato sulla competizione conservatori-laburisti; nell'altra metà (Inghilterra meridionale e aree rurali e suburbane) quello imperniato sulla competizione conservatori- $\mathrm{Al}$ liance. La portata della sconfitta elettorale del Labour nel 1983 non ha l'eguale nella sinistra europea, tranne il caso del partito comunista francese. Le elezioni del 1987 - terza sconfitta elettorale consecutiva per il Labour - sebbene contrassegnate da una lieve ripresa (Labour: 30,8\%; Alliance: 22,5\%; Conservatori: $42,3 \%$ ), non cambiarono di molto la geografia elettorale emersa nel 1983 e le prospettive del Labour come alternativa di governo. Per gran parte degli anni ottanta, insomma, il Labour è stato dato per spacciato. Il suo destino appariva, a fronte di una relativa stabilità del voto conservatore e di una egemonia parlamentare conservatrice, quello di un permanente «status di minorità» (Harrop 1988, 34).

La sua credibilità come forza politica nel lungo periodo appariva come definitivamente compromessa (Sarvik e Crewe 
1983; Butler e Kavanagh 1984 e 1988; Robertson 1984). Sul versante non delle sue basi elettorali e del suo radicamento sociale-geografico, ma su quello politico-istituzionale il pessimismo sulla sua capacità di tenuta era ancora maggiore. Dilaniato dalle lotte di fazione interne per un decennio (dalla metà degli anni settanta fino alla metà degli anni ottanta almeno), diretto tra il 1979 e il 1983 dalla debole leadership di M. Foot, crollato nella membership (da 666.091 iscritti individuali nel 1979 a 348.156 nel 1980), anchilosato nel funzionamento della sua macchina organizzativa, radicalizzatosi in una maniera tale tra il 1979 e il 1983 da mutare il tradizionale equilibrio di potere all'interno e da assumere politiche estremistiche che rompevano il suo rapporto tradizionale con il Cabinet-government (McKenzie 1963 e 1982) e il modello vincolante della competizione bipartitica, muto, incapace, confuso nella sua funzione di opposizione ufficiale, tanto da raggiungere alti tassi di assenteismo dei suoi membri nelle sedute parlamentari (Kavanagh 1989; Riddel 1989), il partito sembrava votato «al più lungo suicidio politico mai verificatosi nella storia» (Mitchell 1987). Fino al 1987 esso era ormai un «partito-ghetto» (Massari 1989) e un partito «vecchio».

\section{Crisi del partito laburista e del bipartitismo}

Vale la pena di indicare sommariamente alcune implicazioni che la percezione diffusa del declino ritenuto irreversibile del partito laburista comportava sul piano più generale del sistema partitico-istituzionale.

Diversamente dagli anni sessanta e settanta, quando la crisi del bipartitismo poteva venire attribuita alla lenta erosione elettorale delle basi sociali dei due maggiori partiti, alle trasformazioni sociali, alla comparsa di nuovi soggetti politici (gruppi di pressione in particolare e burocrazia governativa) (Beer 1965 e 1982), all'incrinarsi della tradizionale omogeneità politica (Blondel 1967), all'irrazionalità dell'adversary politics (Finer 1975) e al consumarsi storico del «modello Westminster» (Johnson 1977; Norton 1982; Bogdanor 1983; Jowell e Oliver 1985), gli anni ottanta hanno testimoniato l'intreccio assai stretto tra crisi/declino del Labour e crisi del bipartitismo. Ma, venuto meno nel paese il modello bipartitico, anche il sistema istituzionale viene sottoposto a critica: non si vedono più le ragioni 
per continuare ad avere una costituzione non scritta, un sistema elettorale non proporzionale, il primato dell'esecutivo sul legislativo, governi monopartitici ecc. Come si vede tutta la crisi del sistema politico-istituzionale sembra precipitare e concentrarsi nella crisi del partito laburista, in quanto ciò che sorregge tutta l'architrave del sistema - in una serie di correlazioni stringenti, storicamente sedimentatesi - è il formato bipartitico di questo stesso sistema. Quando uno dei componenti del sistema bipartitico viene meno nelle sue molteplici funzioni (di rappresentanza «nazionale», maggioritaria, di opposizione ufficiale in grado di costituire una alternativa credibile di governo ecc.), anche tutto il resto del sistema istituzionale risulta squilibrato e alla ricerca di un nuovo assetto democratico (Mair 1989; Laver 1989). In questo senso, la crisi/declino del partito laburista non può essere letta semplicemente come una crisi interna o organizzativa o ancora del suo cleavage di classe ma anche come crisi esterna di un sistema politico-istituzionale cui il partito sin dalla sua nascita risulta strettamente connesso. La connessione tra partito e sistema istituzionale la si può ben cogliere a livello della stessa struttura organizzativa e degli stessi assetti di potere interni. La prevalenza del Labour Parliamentary Party (PLP) sugli organi interni ed extraparlamentari di partito (National Executive Committee - NEC -, Conference ecc.) è stata spiegata tradizionalmente con il riferimento alla natura federativa, indiretta e al basso grado, conseguentemente, della sua istituzionalizzazione (Panebianco 1982, 178). La spiegazione è cioè di tipo genetico-organizzativo. Il problema sorge quando si vede che tale preminenza persiste ed anzi aumenta quando il partito, come negli anni cinquanta, sembra avere raggiunto una «centralizzazione di tipo socialdemocratico», un controllo pieno dei propri confini organizzativi e ideologici (Shaw 1988). Il fatto è che una correlazione ben più cogente sembra esserci tra primato del partito parlamentare e regole e vincoli del governo di gabinetto che con il «modello di partito». Nel responsible party government e nel Cabinet-government, infatti, gli eletti - in nome della sovranità del Parlamento - sono responsabili innanzitutto e primariamente verso l'elettorato piuttosto che verso l'organizzazione partitica o gli iscritti di questa. Da questo principio discende il primato del partito parlamentare nel policymaking e nelle attribuzioni decisionali. Il soggetto del party government nel modello Westminster di democrazia rimane, infatti, pur sempre il partito parlamentare e la sua leadership (Calise 
1989). I partiti politici britannici sono stati condizionati, nella loro evoluzione, dalla forza vincolante sui comportamenti della forma di governo parlamentare basata sul primato del Cabinet e del party government (Cox 1987; Massari 1992a). Il partito laburista si adegua sin dall'inizio alle regole e convenzioni istituzionali del sistema (Guttsman 1963), tanto che è problematico inserire il «modello» del partito laburista in quello delle opposizioni «strutturali» 1 .

Se questa correlazione tra partito e sistema istituzionale è corretta, allora spiegazioni della natura o del modello di partito solo svolte in chiave sociologico-genetica (origine, cleavages) o organizzativa non sono sufficienti a cogliere la dimensione anche istituzionale del partito. La funzione prevalente dei partiti britannici è, com'è noto, quella istituzionale di esprimere un governo e una opposizione ufficiale in grado di essere a sua volta un governo alternativo. Questa funzione si impone anche su quelle altre funzioni - come ad esempio quella di rappresentanza - pure essenziali al sorgere di un partito politico, com'è il caso specifico del Labour. Ma se ciò è vero, allora questa specifica funzione istituzionale, «di governo», non può non entrare a far parte di una definizione che voglia essere esaustiva del «modello di partito».

Quando tra il 1979 e il 1983 il partito laburista muta radicalmente la sua struttura di potere interna in nome della democratizzazione ${ }^{2}$ (Massari 1989), il risultato sarà disastroso perché affida di fatto poteri delicatissimi agli attivisti e ai funzionari di base, in assenza di un partito di massa a base individuale in grado di praticare una democrazia interna non contrastante con quella esterna parlamentare (Bochel e Denver 1983; Drucker

\footnotetext{
' In quanto accetta sin dall'inizio le regole e convenzioni del sistema istituzionale, il Labour non svilupperà mai una opposizione al sistema o non avrà mai problemi di integrazione "positiva", giacché - nonostante la sua origine esterna - si dà un assetto di poteri interni congruente e funzionale alla peculiare forma di governo britannica. Semmai, il conflitto non avverrà verso il sistema, ma all'interno del partito extraparlamentare tra le sue diverse componenti e centri di potere (ad esempio tra NEC, Conference e gruppo parlamentare o tra le diverse tendenze). Partito di classe, il Labour non è stato mai un partito della lotta di classe (Cook e Taylor 1980).

2 Tra i mutamenti introdotti: elezione del leader e del deputy-leader da parte di un collegio elettorale, i cui voti sono assegnati per il $30 \%$ ai constituency parties, per il $30 \%$ al gruppo parlamentare e per il $40 \%$ alle unions, e non più al solo gruppo parlamentare; obbligo di reselection per i parlamentari in carica; eliminazione di molti poteri e prerogative tradizionalmente affidati al PLP, come la stesura del Manifesto elettorale e la responsabilità delle politiche in Parlamento (Massari 1989).
} 
1981 e 1983; Geckie e Levy 1988; Kavanagh 1982). Lo spostamento del baricentro di potere dal modello tradizionale - in cui PLP e leadership godevano di una relativa autonomia - alla cosiddetta democrazia di base, praticata solo da un ristretto numero di attivisti, avrà come conseguenza quella di rompere il delicato rapporto tra partito e sistema di governo. Birch noterà, a proposito di queste riforme organizzative, che «sviluppi dentro il Labour Party hanno riaperto domande circa la compatibilità della intra-party democracy con i principi della democrazia parlamentare» (Birch 1985, 77).

Assieme a queste riforme organizzative radicali, passano corrispondenti politiche settarie ed estremistiche. Se si considera anche tutto questo versante politico-istituzionale, allora la crisi del Labour non appare legata solo alle trasformazioni sociali, alla riduzione del lavoro manuale, ai processi di dealignment elettorale e partitico, ma anche a fattori più squisitamente politici. La spiegazione del dealignment (Franklin 1985) non appare pienamente esaustiva. A parte chi nega persino il fenomeno per ciò che concerne il partito laburista (Heath et al. 1985) o lo inserisce in un diverso modello esplicativo, in cui conterebbero di più i cleavages settoriali rispetto a quello classico di classe (Edgell e Duke 1991; Dunleavy e Husbands 1985), la ricerca empirica sul comportamento di voto sembra sempre più concordare sullo stretto intreccio tra fattori sociali e fattori politici attribuibili al partito (che conterebbero in misura almeno pari ai primi).

\section{I fattori politici della crisi laburista}

Ridimensionata la capacità esplicativa dei soli fattori sociologici per spiegare il declino del Labour negli anni ottanta, è, dunque, ai fattori politici (comprendenti anche quelli organizzativi e di rapporto con il sistema di governo) che occorre rivolgere l'attenzione, dando loro un più significativo peso.

I principali di questi fattori possono essere così sintetizzati:

a) incompetenza governativa. I governi laburisti del 1964-70 e del 1974-79 sono stati giudicati fallimentari. Tale giudizio di fallimento evidenziava la crisi del modello socialdemocraticokeynesiano. Negli anni sessanta e settanta divenne sempre più chiaro il conflitto tra gli obiettivi del mantenimento del Welfare State e quelli della crescita economica. I governi laburisti non 
seppero fronteggiare il permanente declino economico del paese - che è poi una delle questioni chiave su cui gli elettori giudicano i partiti al momento delle elezioni - la crescente inflazione, la intensificata conflittualità dei sindacati nelle relazioni industriali, ecc. Essi furono costretti, così, ad operare tagli nella spesa pubblica, a introdurre limiti alla crescita salariale, l'effetto dei quali fu quello di abbassare gli standard di vita di molti elettori dello stesso Labour (Coates 1980; Hennessy e Seldon 1987). Il bilancio fallimentare di queste esperienze governative fu attribuito alle politiche della destra socialdemocratica che aveva governato il partito sin dagli anni cinquanta. Questo senso di incompetenza governativa spiegherebbe la sconfitta elettorale del 1979 e la vittoria dei conservatori guidati da un leader - Margaret Thatcher - che, rompendo la tradizionale consensus politics (Kavanagh e Morris 1989), si presentava con un programma radicale improntato rigorosamente al neo-liberismo.

b) Partito estremista. La crisi del modello socialdemocratico e il discredito della dirigenza «di destra» crearono lo spazio perché la sinistra interna divenisse, dopo il 1979, la forza dominante dentro il partito. Già durante gli anni settanta essa aveva accresciuto la propria presenza organizzativa e la propria influenza dentro i sindacati, finendo con il conquistare il controllo del NEC. Tra il 1979 e il 1983 il partito fu così spostato radicalmente a sinistra, sia organizzativamente (con le riforme cui si è già accennato), sia politicamente (con la proposizione di politiche più «socialiste»), sia ideologicamente (dal rilancio della lotta di classe all'esaltazione dei temi delle varie minoranze emarginate e/o radicali) (Kogan e Kogan 1983; Kavanagh 1982).

I risultati dello spostamento a sinistra del partito furono non solo meno positivi di quelli ottenuti sotto la destra, ma si rivelarono disastrosi: il partito fu dilaniato dalle lotte di fazione interna; subì una scissione a destra nel 1981 (l'ultima scissione era avvenuta nel lontano 1932); patì nel 1983 la più cocente sconfitta elettorale dal 1918.

Da notare che nelle elezioni del 1983 il tasso di disoccupazione raggiungeva la cifra di tre milioni: era la prima volta che un governo conservatore vinceva le elezioni nel contesto di una situazione sociale potenzialmente favorevole alla sinistra (disoccupazione massiccia, licenziamenti, disagio sociale diffuso ecc.). Naturalmente contarono anche altri fattori, come la guerra delle Falklands, ma su tutti si stagliava l'immagine del partito labu- 
rista come un partito dominato dall'estremismo, dalla faziosità e dal settarismo.

c) Dipendenza del partito dai sindacati e organizzazione sclerotizzata. Il Labour ha continuato ad essere un partito indiretto e dipendente fondamentalmente dai sindacati, con ciò precludendosi quella capacità di innovazione e quella libertà d'azione che $\mathrm{i}$ partiti diretti possono esercitare in più grande misura (Mair 1983). Negli anni settanta la conflittualità e i molti poteri/privilegi dei sindacati sono emersi come uno dei problemi più acuti del paese. Non a caso uno dei principali punti di attacco dei governi della Thatcher negli anni ottanta fu proprio il potere del sindacato (Hanson 1991). In tutti i sondaggi di opinione dell'ultimo decennio la dipendenza dai sindacati del partito laburista è indicata come uno dei dati più negativi. Ancora oggi i conservatori attaccano i laburisti, nella propaganda elettorale, con lo slogan: Who runs Labour? La dipendenza dai sindacati, inoltre, ha effetti negativi sia sul piano del processo decisionale interno - con il sistema del block vote i sindacati determinano l'agenda politica della Conference - sia su quello dell'immagine complessiva del partito come partito strettamente della classe operaia e legato alle politiche del collettivismo classista (nazionalizzazioni, alte tasse ecc.). Nel passato sia Gaitskell nel 1959, sia Wilson nel 1964 avevano tentato - ma senza successo - di modificare la natura del partito, trasformandola da quella strettamente di classe a quella di partito «nazionale» (Hamilton 1989; Howell 1976; Smith 1991).

d) Deficit di rappresentanza. L'incapacità del partito, a seguito di tutti questi fattori, si manifestò sul terreno della rappresentanza sociale e culturale di tutto lo spettro dell'area di sinistra progressista, compresi quei settori della stessa classe operaia che si rivolgevano sia ai conservatori sia ai partiti di centro. Il risorgere delle fortune e delle prospettive dell'alternativa di centro (liberale e socialdemocratica) testimoniava la rottura nel paese, se non in Parlamento, del sistema bipartitico e l'affermazione di un modello tripartitico nel quale il partito laburista aveva perso il monopolio della rappresentanza sociale della sinistra (Bradley 1981). Solo il sistema elettorale e il radicamento sociale del partito in alcune aree geografiche nascondevano questa realtà.

Dalla somma di tutti questi fattori non poteva che emergere un quadro estremamente negativo per il partito e la sua immagine pubblica. I fallimenti riguardavano tanto il modello social- 
democratico degli anni sessanta e settanta, quanto le realizzazioni della sinistra interna tra la fine degli anni settanta e il 1983; tanto la sua capacità di governo quanto la sua capacità di rappresentanza.

Da questo punto di vista complessivo, il declino organizzativo ed elettorale appariva di lungo periodo e niente affatto limitato al periodo thatcheriano.

Debole sembra, infatti, quella spiegazione secondo cui le tre sconfitte elettorali consecutive del partito laburista $(1979,1983$ e 1987) sarebbero da attribuire alla offensiva antisocialista della Thatcher. In questa spiegazione «politicista» la relazione causeeffetti appare rovesciata: è assai più fondata la tesi contraria, secondo cui sarebbe stata proprio la spaccatura dell'opposizione tra vecchio Labour e nuovo centro a permettere al blocco thatcheriano di prevalere.

\section{Il processo di innovazione}

Come il declino di un partito di massa non è quasi mai un evento improvviso e immediato, ma è l'accumulazione di medio-lungo periodo di trend sociali negativi e di errori e fallimenti politici, così il suo rinnovamento - posto che la leadership si proponga di perseguirlo coerentemente - non può essere altrettanto rapido. Non basta volere o proclamare il rinnovamento, occorre «implementarlo» tanto nell'ambiente esterno (opinione pubblica, elettorato), quanto in quello interno. Le due operazioni sono ovviamente correlate. Il partito non sarà percepito all'esterno come rinnovato se tale percezione non include anche il rinnovamento interno. Ma è noto come, quanto più i partiti sono a «struttura pesante» (e lo sono certamente i partiti di massa a base operaia), tanto più presentano rigidità organizzative e ideologiche. Tali rigidità interne sono il principale ostacolo all'innovazione. E difficilmente la leadership di un partito a struttura pesante può ignorare i rigidi vincoli che gli provengono dall'interno. Si tratta, allora, per la leadership di convincere i membri e gli apparati interni della necessità del cambiamento. E si tratta, inoltre, del fatto che il cambiamento sia percepito effettivamente come tale dall'esterno. Tutto questo implica varie cose. Prima di tutto che le vecchie e impopolari (all'esterno) politiche siano realmente cambiate nella direzione che può essere compresa e accettata dall'elettorato. In se- 
condo luogo, il cambiamento delle politiche non basta, esso è solo una parte del processo.

Occorre comunicare tale cambiamento. E comunicarlo tramite una strategia comunicativa, per così dire, a doppio binario, interno ed esterno, la cui coerenza e congruenza può essere garantita solo da una leadership sufficientemente radicata nel e appoggiata dal partito, sufficientemente motivata all'innovazione, adeguatamente informata sui trend sociali e sugli orientamenti dell'opinione pubblica. Può accadere, infatti, che un messaggio accettabile all'esterno non sia tale all'interno e viceversa. In ogni caso, occorre che non ci sia una «doppia verità», una per l'interno e l'altra per l'esterno.

Né ci possono essere messaggi contraddittori provenienti dall'interno e diretti verso l'esterno. Il messaggio deve essere univoco, chiaro, comprensibile. Ossia il partito deve essere unito e sembrare tale. Ciò, detto in altri termini, implica che la leadership abbia il pieno controllo di questo e che non possa essere smentita o contraddetta da questo o quel settore dell'organizzazione. Tutte queste modalità dell'innovazione in parte provengono dai vincoli interni dell'organizzazione di un partito a struttura pesante, in parte dai vincoli che il tipo di competizione politica - nel modello di democrazia maggioritaria britannica - impone al partito che intenda «adattarsi» a tale modello.

Contenuto delle politiche, strategia comunicativa, ruolo della leadership, nuovo modello di partito sono tutti ingredienti di primaria importanza nei processi di «adattamento», «controllo», innovazione dei partiti. Sono questi ingredienti che si ritrovano al centro del processo di rinnovamento del partito laburista. Vale la pena di evidenziare analiticamente ognuna di queste dimensioni dell'innovazione, dando per scontato il loro carattere processuale e interconnesso.

\section{L'uso della comunicazione politica e delle scienze sociali}

La comunicazione politica. Dopo il dramma del «terribile risveglio della mattina del 10 giugno 1983», quando si appresero i disastrosi risultati elettorali del partito, i peggiori dal 1918, la nuova leadership di Kinnock - eletta nell'autunno di quello stesso anno - era estremamente determinata a non far ripetere più al partito tale esperienza. Per questo era psicologicamente $\mathrm{e}$ politicamente disposta alle innovazioni anche più radicali pur di 
rendere nuovamente electable e governmental il partito. L'imperativo elettorale, quello di riguadagnare il consenso tra gli elettori, fu, perciò, l'imperativo primario. Necessariamente la comunicazione politica ebbe un posto di primissimo piano nel perseguimento di tale imperativo.

Nel 1985 fu formata la Shadow Communications Agency, un organismo composto da professionisti dei mass media (pubblicisti, giornalisti televisivi, manager delle comunicazioni, esperti in sondaggi ecc., tra $\mathrm{i}$ nomi divenuti famosi quelli di Philip Gould e Peter Mendelson). Contestualmente fu creato un settore interno per le Campaigns and Communications. Al di là di quello che possono dire queste nuove strutture, ciò che fu importante era la piena accettazione della centralità della comunicazione politica all'interno del processo di rinnovamento del partito. Ancora più importante era la nuova filosofia comunicativa che venne affermata da queste strutture e accettata dalla leadership. Non si trattava, infatti, semplicemente di rivolgersi ad un'agenzia esterna per curare l'immagine del leader e del partito, come ad esempio facevano i conservatori che si servivano della famosa Saatchi \& Saatchi. Ciò era possibile per i conservatori perché - a parte la maggiore disponibilità finanziaria - il partito extraparlamentare delegava completamente alla leadership la formulazione e presentazione delle politiche e dell'immagine pubblica. Non pretendeva, cioè, almeno formalmente alcuna partecipazione nel processo decisionale del policy-making. In un partito come quello laburista, al contrario, le strutture organizzative interne hanno difficoltà a rapportarsi ai processi della comunicazione politica, giacché proprio queste strutture costituiscono i principali canali e fonti dell'informazione e della comunicazione. Soprattutto, queste strutture sono gelose di questa loro funzione, da non essere facilmente disposte a delegarla a centri esterni. La nuova filosofia comunicativa, propugnata dagli uomini della Shadow Communications Agency, implicava, al contrario, una radicale revisione dell'approccio tradizionale al policy-making e alla «vendita» dell'immagine.

Questa revisione sollecitava, essenzialmente, il passaggio da un processo di formulazione delle politiche basato sulla democrazia interna di partito - quindi sui comitati interni - a un processo basato sulla comunicazione con l'ambiente esterno nel determinare le posizioni del partito. I temi delle campagne di mobilitazione e della propaganda, ad esempio, non dovrebbero 
più essere determinati dagli attivisti di base, o scaturire dal loro universo culturale di riferimento, ma dovrebbero scaturire dalla capacità di esercitare influenza elettorale attraverso i mass media (Hughes e Wintor 1990).

La prima priorità, per questa nuova filosofia della propaganda di partito, era allora quella di guardare anzitutto alle opinioni dell'elettorato, rilevabili non più attraverso i metodi tradizionali delle campagne politiche, ma attraverso le varie tecniche quantitative e qualitative dei sondaggi di opinione.

Ciò richiedeva non solo l'accettazione da parte delle strutture interne di tecniche e culture sempre guardate con sospetto oltretutto perché espropriavano funzioni proprie dell'organizzazione - o accettate solo strumentalmente, ma anche la disponibilità all'«ascolto» delle risposte. Il che è cosa assai più difficile per $i$ partiti socialisti, cresciuti in una visione pedagogica del rapporto partito-classe-elettorato.

La campagna elettorale del 1987 costituì il campo di applicazione e la prima prova di questo nuovo approccio all'uso della comunicazione politica. La campagna, in effetti, riscosse un riconoscimento unanime.

Secondo tutti gli osservatori e gli esperti, la campagna del Labour - che fu definita di «stile presidenziale» o all'americana - fu tecnicamente superiore a quella dei conservatori, gestita per la parte dell'immagine da Saatchi \& Saatchi. Ma il successo tecnico della campagna non si tradusse in un successo politico come sperato, anche se non va sottovalutato che il partito superò, forse definitivamente, il pericolo di essere scavalcato dai partiti dell'Alliance.

La dura, ma salutare, constatazione fu che le tecniche e l'innovazione nella comunicazione politica (ossia il rinnovamento solo simbolico o «cosmetico», come accusavano i conservatori) non possono sostituire il contenuto delle politiche. Le proposte programmatiche inserite nel Manifesto elettorale laburista del 1987 erano ancora, sia pure ammorbidite, quelle del 1983. Ma i primi ad esserne consapevoli erano proprio gli esperti della Shadow Communications Agency. La loro profonda convinzione era che non bastasse cambiare la confezione o la presentazione del messaggio, ma occorresse cambiare anche il prodotto, ciò che si offriva. Il loro tentativo di rivoluzione mass-mediologica prima del 1987 aveva incontrato limiti insormontabili nella durezza degli apparati e delle strutture. L'innovazione comunicativa era stata legittimata - anche dal successo tecnico della campagna 
del 1987 - ma mancava ancora l'innovazione politica vera e propria.

L'uso delle scienze sociali. Se l'esigenza primaria della leadership - ma avvertita anche da vasti settori del partito e dei sindacati - era quella di rendere il partito electable e governmental, il problema era non solo quello di conquistare il consenso dell'elettorato, ma soprattutto di conoscerne le aspettative, i valori, gli atteggiamenti. Si trattava, cioè, di conoscere e capire la società britannica al di là dei filtri distorcenti delle organizzazioni interne del partito, utilizzando più obiettivi e moderni strumenti di conoscenza. Questa opportunità venne al partito dallo stesso gruppo di uomini delle comunicazioni.

Una vasta ricerca sull'opinione politica in Gran Bretagna, condotta durante i mesi della campagna elettorale del 1987, fu diretta dalla Shadow Communications Agency, utilizzando sia altre ricerche esistenti sia promuovendo analisi in proprio. L'Agency aveva formato un gruppo di studio - in vista della stesura di un rapporto ad uso della dirigenza - formato da specialisti: da suoi stessi membri (Mendelson, P. Gould, Hewitt), da R. Jowell del British Social Attitudes Survey, da A. McIntosh, membro laburista dei Lords che dirigeva una società di analisi di mercato, da A. Shaw e J. Curtice dell'Università di Liverpool (questi ultimi intrapresero un'analisi di tutti i dati disponibili sulle elezioni degli ultimi venticinque anni).

L'analisi dell'opinione politica, attraverso metodi quantitativi e qualitativi, era collocata all'interno dell'analisi più generale dei cambiamenti sociali che la Gran Bretagna stava attraversando e che si sarebbero realizzati, prevedibilmente, nei successivi dieci anni nella sfera dell'economia, degli stili di vita e delle aspirazioni individuali. Il rapporto conclusivo, noto con il titolo Labour and Britain in the 1990s, presentato nel novembre 1987 ad una riunione congiunta del NEC e del governo-ombra, conteneva delle conclusioni politicamente esplosive rispetto alle idee prevalenti.

Queste conclusioni possono essere così riassunte:

a) i valori «thatcheriani», come la fede nella superiorità del libero mercato e del settore privato, non erano riusciti ad avere una reale presa sulla popolazione, ma - rovescio della medaglia -

b) molte delle cose a cui la gente aspirava, come la proprietà individuale della casa, erano identificate strettamente con la politica thatcheriana; 
c) il divario di genere (il rapporto tra voti femminili e maschili) si stava restringendo nell'elettorato laburista. Per vent'anni i conservatori avevano avuto la guida nell'elettorato femminile; ora il Labour l'aveva assunta tra la fascia giovane, ma questo era anche il risultato di un restringimento del voto dell'elettorato maschile verso il partito;

d) l'analisi condotta da Show e Curtice mostrava che i cambiamenti nella struttura di classe (declino numerico della classe operaia e aumento del ceto medio) avrebbero da soli prodotto una diminuzione del voto pro-Labour del 6\% dal 1964 al 1987. In realtà, il declino del sostegno elettorale in questo periodo era stato del $13,3 \%$, suggerendo, dunque, che non tutte le perdite potevano essere attribuite al cambiamento sociale e, se così era, queste si potevano recuperare con relativa facilità;

e) i laburisti registravano una fedeltà di voto tre volte inferiore a quella dei conservatori;

f) il partito era considerato all'esterno come dominato da una cultura politica estranea (alien) e da una cultura maschile intenta solo a prescrivere come la gente dovrebbe vivere piuttosto che rendere possibile che gli individui facessero le loro scelte in proprio;

g) la campagna del 1987 era stata persa in partenza perché c'era un senso di crescente prosperità nelle aree meridionali del paese (determinanti ai fini elettorali) e questa prosperità veniva attribuita ai conservatori;

h) le nazionalizzazioni non erano state appoggiate da più di un terzo dell'elettorato sin dal 1964;

i) il $56 \%$ della gente credeva che sotto un governo laburista una crisi economica sarebbe stata probabile;

1) non più di un quarto dell'elettorato, negli ultimi cinque anni, si sentiva particolarmente vicino alla politica del partito nel campo della difesa (consistente nella proposta del disarmo nucleare unilaterale);

m) tra i fattori principali della disaffezione elettorale verso il partito (comprese fette dell'elettorato già in precedenza $\mathrm{La}$ bour) c'era, oltre alla politica della difesa, l'immagine di un partito estremista, diviso da lotte di fazioni interne, privo di una forte leadership personale, controllato dai sindacati, incompetente sul piano economico, legato ad una politica di alte tasse;

n) ultima constatazione: il partito aveva perso voti in tutte le classi sociali.

Era questa una diagnosi precisa dei limiti politico-culturali 
del partito, così come una radiografia esatta dei cambiamenti sociali e del loro impatto sulle aspirazioni della gente. Il rapporto indicava non solo i temi della revisione politica ma anche l'approccio con cui affrontarla: se si voleva riconquistare l'elettorato, l'imperativo era quello di partire anzitutto dalle aspirazioni della gente e poi filtrare, selezionare ed aggregare le loro domande.

Pur senza essere assunto come base ufficiale del processo di revisione politica - che sarebbe iniziato ufficialmente nel 1987 con il lancio della Policy Review - di fatto il rapporto costituì l'agenda politica della direzione del mutamento nelle politiche e dell'approccio con cui affrontarlo. Tale approccio implicava l'inseparabilità del mutamento politico dall'imperativo della comunicazione e delle sue tecniche. Il lavoro svolto dagli esperti delle comunicazioni e delle scienze sociali aveva posto una serie di problemi, di impostazioni e di valutazioni che sarebbe stato difficile ignorare.

\section{La Policy Review e il cambiamento delle politiche}

La Policy Review ${ }^{3}$ - per le molteplici finalità che si poneva, per l'ampiezza dei temi trattati, per la durata temporale che richiedeva (tre anni) e la scansione in fasi distinte dell'intero processo, per i metodi di lavoro proposti - ha costituito un progetto di rinnovamento così complesso e organico che non ha l'eguale nei partiti socialisti europei, a parte la trasformazione da partito marxista a partito popolare della socialdemocrazia tedesca nel Congresso di Bad Godesberg (1959).

Le finalità che soggiacevano all'intero pacchetto di proposte erano di vario tipo. Anzitutto, finalità elettorali: restringere il

${ }^{3}$ L'idea iniziale della Policy Review come progetto organico, di medio periodo, con una sua metodologia di lavoro precisa, non fu una iniziativa della leadership - che però la sostenne politicamente e con convinzione -, ma di un dirigente di uno dei più grossi sindacati affiliati, Tom Sawyer della NUPE, e presidente di uno dei comitati più importanti della direzione del partito (Home Policy sub-Committee). Egli presentò nel settembre 1987 un paper intitolato An Approach to Policy-Making all'Home Policy Committee che, approvato anche dal NEC e dallo Shadow Cabinet, divenne la base di tutto l'esperimento. Il testo finale della Policy Review viene presentato pubblicamente il 18 maggio 1989 sotto il titolo generale Meet the Challenge. Make the Change. A New Agenda for Britain. Final Report of Labour's Policy Review for the 1990s, London, Labour Party, e approvato formalmente dall'intero partito nella Conference di Brighton del 2-6 ottobre 1989. 
divario tra le politiche del partito e le posizioni dell'elettorato. Come si specificava nella presentazione del progetto, questo implicava convincere l'elettorato della bontà delle proposte del partito da una parte, ma dall'altra implicava un andare verso le sue esigenze e domande, mettendo in conto, a tal fine, una revisione profonda delle politiche. $\mathrm{Ma}$ il riferimento alle necessità elettorali del partito come asse centrale della revisione programmatica, non implicava in ogni caso l'abbandono dei valori socialisti del partito laburista. L'aggiustamento progressivo delle politiche - che è bene sottolineare è pensato e voluto come un processo graduale, senza rotture improvvise - è inserito sempre all'interno di una più aggiornata ridefinizione dei valori e dei fini socialisti. Emerge qui una significativa tensione tra necessità del cambiamento delle politiche tradizionali e necessità di giustificare tale cambiamento alla luce dei valori tradizionali, ma ridefiniti e aggiornati ${ }^{4}$.

In secondo luogo, la Policy Review era intesa come un modo di rivitalizzare la partecipazione interna dei membri, coinvolgendoli in una vasta e non occasionale discussione sul programma e nell'esercizio democratico del policy-making. Questa seconda finalità interna era oltretutto importante e necessaria se si voleva colmare il divario tra la membership e l'opinione pubblica e ritrovare un largo consenso interno di sostegno a politiche di partito che fossero più popolari nell'elettorato. L'impostazione, infatti, di un duplice canale comunicativo all'esterno e all'interno faceva sperare che i due canali di partecipazione si potessero incontrare, dando luogo ad una sinergia tra elettorato e membership.

Infine, un'altra finalità - ma non meno importante - aveva uno scopo simbolico: già nel mettere in piedi un processo di revisione politica si dava l'impressione di un partito desideroso di cambiare, di rinnovarsi, di aggiornarsi.

La durata del processo di revisione fu programmata in tre fasi: a) la prima fase avrebbe dovuto produrre «una valutazione dei valori, degli obiettivi e dei principali temi delle politiche», che sarebbe stata considerata dalla Conference del 1988; b) la seconda fase, da concludersi con la Conference del 1989, sarebbe stata dedicata alla formulazione delle proposte sulle più importanti politiche programmatiche; c) l'ultima fase doveva esse-

4 Tale ridefinizione è esposta in un documento di partito, a firma di Kinnock e di Hattersley, Democratic Socialist Aims E Values, London, Labour Party, 1988. 
re finalizzata alla decisione finale sul programma generale e concentrarsi sulla promozione di questo programma in tempo per le elezioni politiche.

Da notare che l'intero processo era inteso come aperto, non predeterminato da opzioni precostituite dalla leadership o da altri. Era questa una condizione per ottenere che tutto il partito si impegnasse in un esercizio democratico di policy-making basato sull'esigenza del rinnovamento.

I temi da affrontare nella revisione coprivano pressoché tutta l'area delle questioni politiche facenti parte del programma di un partito, con l'esclusione dei problemi relativi al modello organizzativo e alla democrazia interna del partito. Ogni tema politico doveva essere affrontato da uno specifico gruppo di lavoro. Furono formati ben sette gruppi di lavoro sui seguenti temi:

\section{Productive and Competitive Economy;}

2. People at Work;

3. Economic Equality;

4. Consumers and Community;

5. Democracy for the Individual and the Community;

6. Physical and Social Environment;

7. Britain in the World.

La tematizzazione indicava chiaramente come, accanto a settori tradizionali, la revisione delle politiche includesse terreni nuovi d'impegno (ambiente, i diritti dei consumatori e degli individui) che stavano ad indicare la volontà di uscire fuori dalla tradizionale impostazione collettivista e industrialista del Labour. Tuttavia, nuove mediazioni e compatibilità dovevano essere trovate tra libertà individuali e comunità, tra il mercato e lo Stato, tra le esigenze della crescita economica e la salvaguardia dell'ambiente naturale e sociale. Era cioè posto come un problema, come un campo di tensioni tra dimensioni conflittuali, ciò che precedentemente era negato in nome di valori ideologici precostituiti e unidimensionali (collettivismo, statalismo ecc.).

Quanto ai metodi di lavoro, la Policy Review avrebbe continuato e potenziato la pratica delle riunioni congiunte tra NEC e governo-ombra. Ciascun gruppo di lavoro doveva essere coordinato da due esponenti di questi due organi. Oltre a membri del NEC e del governo-ombra, i gruppi dovevano essere composti da leaders dei sindacati più rappresentativi e da un ristret- 
to numero di «esperti». Un più largo numero di esperti, provenienti dal mondo accademico, dai gruppi di pressione e dai sindacati, furono coinvolti in una varietà di modi: dalla richiesta di pareri e analisi scritti ad audizioni, dalla partecipazione a seminari all'inserimento in sottogruppi.

Il lavoro dei gruppi doveva svolgersi in stretto collegamento con il mondo delle competenze e con i centri e le fonti delle analisi, delle informazioni, dei dati. Ciascun gruppo - formato da 7-10 membri, ma con numerosi sottogruppi e gruppi di studio - doveva, inoltre, tenere pieno conto dello stato esistente della pubblica opinione. Un flusso continuo di informazioni era garantito a ogni gruppo dal Political Intelligence Officer del partito, che aveva la responsabilità di produrre rapporti che sintetizzavano i dati delle ricerche quantitative esistenti sul mercato, e dalla Shadow Communications Agency, specializzata in ricerche qualitative, interviste in profondità con campioni selezionati di gruppi sociali che riguardavano principalmente gli atteggiamenti verso le politiche, i valori e le forme del linguaggio politico.

Fu stabilito un meccanismo per il coordinamento del lavoro dei vari gruppi tramite un Campaign Management Team al vertice (presieduto dallo stesso Sawyer e composto dal segretario generale del partito, da rappresentanti del Leader Office, dal coordinatore del governo-ombra e da altri funzionari degli uffici centrali del partito). Tutti i gruppi e il coordinamento generale dovevano avere incontri regolari e programmati. Via via che le prime stesure dei rapporti dei vari gruppi erano pronte - ma anche nella stessa fase della formulazione - tutto il partito, al livello di base, cioè nei collegi, doveva essere mobilitato in discussioni, convegni, seminari (per tutte queste informazioni, oltre ai vari documenti di partito elencati in bibliografia, cfr. Shaw 1989; Seyd 1990; Hugh e Wintour 1990; Smith 1991).

Se questo era il disegno complessivo di come avrebbe dovuto funzionare la Policy Review, va, tuttavia, riconosciuto che non tutto di questo disegno si è realizzato. Riguardo all'obiettivo di aprire canali comunicativi verso l'esterno e verso l'interno, il quadro dei risultati finali è, infatti, abbastanza controverso.

La grande campagna di «Ascolto» lanciata verso l'opinione pubblica sin dalla seconda metà del 1987, Labour Listens, - come parte integrante del processo della Policy Review non ha dato $\mathrm{i}$ risultati sperati in termini di reale capacità di 
mobilitazione in tutto il paese, sicché è stata progressivamente messa da parte (Shaw 1989).

Così anche la mobilitazione interna - soprattutto nella fase di partecipazione alla consultazione sulle politiche - non è risultata facile, giacché sono venuti in evidenza i difetti dei constituency parties nell'affrontare compiti e modalità di partecipazione democratica cui per tradizione non erano preparati. Le attività di queste organizzazioni di base erano di solito l'assolvimento di compiti amministrativi o le lotte ideologiche interne di fazione. I singoli gruppi di lavoro hanno avuto anche un funzionamento e una qualità differenziati, a seconda della capacità e dell'impegno dei membri coinvolti (Shaw 1989).

Nonostante queste e altre critiche che possono essere rivolte, la Policy Review ha conseguito, tuttavia, il suo principale obiettivo, quello di cambiare e aggiornare le politiche del partito in modo tale da riconquistare la fiducia dell'elettorato. A conclusione di tutto il processo di revisione delle politiche, conclusosi alla fine del 1989 e messo a punto in una serie di documenti successivi, il Labour Party si presenta al pubblico come un partito ormai completamente nuovo, perlomeno sotto il profilo programmatico.

Vale la pena di indicare $\mathrm{i}$ casi più significativi e universalmente riconosciuti di revisione delle politiche:

- la politica della difesa è stata completamente capovolta. Dall'approccio «unilaterale» e pacifista, prevalente fino al 1987 e non intaccato fino al 1989, il partito è passato ad un approccio «multilaterale», cioè alla decisione di affrontare i problemi del disarmo nucleare nel contesto di negoziati multilaterali e non con misure unilaterali, pienamente rispettoso della solidarietà occidentale e dei vincoli a questa connessi (dalla NATO al rapporto con gli USA). Che il cambiamento non fosse di facciata - come accusavano i conservatori - lo provò la vicenda della Guerra del Golfo nel 1991. In quell'occasione la leadership, Kinnock e tutto il team dell'opposizione parlamentare, sostenne le decisioni interventiste del governo conservatore (una volta approvate dalla maggioranza del Parlamento). Presso l'opinione pubblica il partito apparve pienamente «occidentale» e del tutto in linea con il modello dell'opposizione responsabile. Dopo questa prova, l'accusa al Labour di «non volere difendere la Gran Bretagna» ha perso di significato e di rilevanza;

- il vecchio impegno programmatico per un vasto programma di nazionalizzazioni e di avversione al mercato - ribadito 
ancora nel Manifesto elettorale del 1987 - è stato abolito. Ora il partito dichiara di accettare in pieno un approccio positivo all'economia di mercato, pur all'interno di una visione sociale e comunitaria. Con l'accettazione critica del mercato si riconoscono tutte le compatibilità economiche, nazionali e internazionali (come quelle derivanti dall'adesione alla Comunità europea), e le priorità derivanti dall'imperativo di rendere l'economia britannica, in declino da un lungo periodo, competitiva e produttiva;

- il partito non è più antieuropeo, come ancora fino al 1987 , ed è un sostenitore più convinto degli stessi conservatori della prospettiva europea, pur permanendo ambiguità circa la reale posizione verso l'obiettivo politico di una unità politica intesa come federazione di Stati. Ma in ciò il Labour condivide tutte le perplessità e le obiezioni della società britannica, essendo l'Europa una issue trasversale, che non segue linee partitiche (Massari 1990b);

- il partito non intende più ripristinare - una volta al governo - la legislazione sindacale cancellata dai governi thatcheriani, riconoscendo che molta di questa precedente legislazione conteneva privilegi corporativi o anacronistici. Solo gli aspetti più attinenti alla sfera delle libertà del sindacato saranno rivisti (come la possibilità da parte del governo di confiscare $\mathrm{i}$ beni del sindacato in caso di scioperi non autorizzati).

Che la Policy Review abbia cambiato profondamente l'immagine complessiva del partito non è messo in dubbio da alcuno in Gran Bretagna (a cominciare dai mass media). Può essere messa in dubbio la validità delle nuove politiche, ma questo fa parte del dibattito politico. I conservatori, come anche alcuni settori intellettuali e politici della sinistra più larga, accusano il partito laburista di elettoralismo, di trasformismo per bramosia di potere o di moderatismo.

Sul piano della ricerca, invece, si discute se la Policy Review sia il segno dell'adeguamento al thatcherismo o comunque l'accettazione di temi propri a questo (Jenkins 1989; Kavanagh 1990; Hall 1985; Jessop 1987) - che è l'interpretazione prevalente - oppure se non sia il ritorno al revisionismo socialdemocratico aggiornato di Gaitskell e Crosland (Smith 1991). 


\section{Il rafforzamento della leadership e del partito parlamentare}

L'intero progetto di recupero elettorale e di riconquista della credibilità come partito di governo richiedeva necessariamente un rafforzamento del ruolo politico e personale della leadership e un rilancio della centralità dell'opposizione parlamentare e delle sue strutture. Entrambe queste caratteristiche - forte leadership personale e ruolo decisionale del partito parlamentare - fanno parte integrante del modello Westminster di democrazia. Come Cabinet government, il modello richiede la centralizzazione dell'autorità politica nel leader e il pieno controllo del partito da parte di quest'ultimo. Come party government, il modello si è originato e sviluppato assegnando al partito parlamentare il primato decisionale. In entrambi i significati si richiede un partito del leader, un partito unito, un partito disciplinato, oltre che programmatico. Nella competizione elettorale queste caratteristiche sono importanti. Il partito, cioè, deve adeguarsi ai vincoli e agli attributi che il sistema istituzionale gli pone, pena sanzioni da parte dell'elettorato e dell'opinione pubblica. Il partito laburista, a causa di una configurazione della struttura decisionale interna altamente pluralistica e frammentata tra diversi centri di potere, ha sempre sofferto di un deficit di leadership personale rispetto ai conservatori. In più il controllo dell'intero partito da parte della leadership è condizione dell'unità del partito. E senza unità nel partito non si può aspirare - in un sistema di one-party government - al ruolo pieno di partito di governo.

Il nuovo metodo di elezione del leader nel partito laburista, introdotto nel 1981 e messo in pratica per la prima volta nel 1983 con l'elezione di Kinnock, ha in parte modificato la sua posizione di potere. In quanto eletta sulla base di una legittimazione più ampia e rappresentativa del partito, la leadership si è obiettivamente rafforzata. Se non è più sufficiente, infatti, avere la fiducia del solo PLP ma anche quella di componenti più ampie e significative del partito, ciò pone la leadership in grado di controllare meglio, una volta eletta, tutta la macchina partitica. $\mathrm{E}$ il controllo del partito costituisce la precondizione per potere esercitare un ruolo di innovazione piuttosto che di mediazione, com'è richiesto da una struttura di potere pluralistica. Tuttavia, questa è solo una potenzialità, occorrendo per il suo verificarsi un effettivo consenso di tutte le componenti del partito sulle regole e sulla direzione del cambiamento. La vicenda della leadership di Kinnock conferma questa dinamica. 
Tra il 1983 e il 1987 la volontà della leadership di introdurre innovazioni si scontrò più di una volta, e su questioni importanti, con la rigidità e la resistenza al cambiamento da parte del partito. Quando nel 1984, ad esempio, la leadership propose un progetto consistente nell'aumento del potere degli iscritti rispetto a quello degli attivisti, sostituendo la delegatory democracy con la membership democracy (Seyd 1990), la Conference bocciò tale proposta. La pesante sconfitta insegnò al leader che se voleva continuare il processo di rinnovamento doveva conquistarsi e assicurarsi un appoggio maggioritario tra le diverse componenti del partito. Gli sforzi maggiori furono rivolti perciò alla costituzione e consolidamento di una larga coalizione di sostegno alla leadership, fondata sull'area della sinistra moderata e sulla destra interna del partito e di quella sindacale (che nel frattempo aveva riguadagnato il terreno perduto nel passato). L'innovazione, cioè, era inseparabile dal sostegno interno e da un processo di spostamento convinto del partito sulle posizioni della leadership. Tale spostamento, ovviamente, fu favorito dalla terza sconfitta elettorale consecutiva nel 1987, che in certo modo costringeva al cambiamento, pena l'irreversibilità del declino. È per questo che il processo di innovazione si poté dispiegare pienamente e dare i suoi primi frutti solo dopo tale data.

Ma non bisogna dimenticare che l'incubazione del rinnovamento fu iniziata prima, a riprova del fatto che l'innovazione nei partiti di massa è un processo lento, graduale, che non tollera rotture improvvise.

Tra le linee principali di intervento della leadership occorre menzionare - oltre al nuovo uso della comunicazione politica e al sostegno convinto dato alla Policy Review, già visti - almeno altre tre significative questioni: a) la depurazione del partito dalle infiltrazioni estremistiche e b) una nuova relazione tra gli organi dirigenti del partito (NEC e i suoi comitati) e quelli del governo ombra, che poneva fine all'antagonismo e all'incomunicabilità reciproci; c) la trasformazione del partito da confederato a partito di massa su basi individuali. Le prime sono state sostanzialmente risolte, la terza si è rivelata - come vedremo più avanti - assai più problematica.

Quanto alla prima, è noto come la presenza di estremisti sia stata una delle maggiori colpe attribuite al partito dall'opinione pubblica e riflesse negativamente sia nelle elezioni che nei vari sondaggi elettorali. L'immagine di un partito diviso, dominato 
dalle lotte di fazione interne e da elementi estremisti non è certo una buona immagine per un partito che pretenda di essere adatto e pronto a governare. Per questo sin dalla Conference del 1985 - in cui ci fu la prima espulsione in massa di elementi dell'organizzazione trotzkista Militant - e sino ad oggi l'azione della leadership è stata di inflessibile lotta all'estremismo, ristabilendo la questione dei confini organizzativi e ideologici del partito e imponendo una disciplina rigorosa, premessa necessaria per il rilancio come partito di governo, non inquinato dalle sinistre estreme. In seguito a questa azione, l'accusa di essere estremista o dominato dagli estremisti ha perso di rilevanza nella competizione politica; semmai il partito può essere accusato di essere eccessivamente moderato. Ma sono accuse che fanno parte del bagaglio della polemica politica.

Quanto alla seconda questione, oltre ad una maggiore interazione tra partito parlamentare e partito extraparlamentare, necessaria all'efficienza complessiva e all'unitarietà dell'indirizzo politico, essa comportava una rivalorizzazione del partito parlamentare e del governo-ombra in particolare.

Tutto il progetto di recupero elettorale e di recupero della credibilità come partito di governo richiedeva, infatti, un rilancio della centralità non solo della leadership ma anche dell'opposizione parlamentare (e delle sue strutture).

A parte il ruolo tradizionale che ha la visibilità dell'opposizione parlamentare nella competizione politica all'interno del sistema bipartitico, recenti innovazioni nel Parlamento hanno accresciuto l'importanza delle performances dei partiti parlamentari. L'introduzione nel dicembre del 1989 della ripresa e trasmissione televisiva in diretta dei dibattiti parlamentari nella Camera dei Comuni ha reso ancora più rilevante, di fronte ad un più vasto pubblico che non quello dei giornali colti, il ruolo del Parlamento come «teatro delle opinioni del paese» $(\mathrm{Ba}$ gehot 1963), e dunque quello del confronto diretto tra governo e opposizione (Mitchell 1989).

Lo stesso Kinnock, proveniente da una tradizione di partito legata a stili comunicativi agitatori, rivolti a colpire la base, a volte persino demagogici, comunque più $\mathrm{da}$ tribuno popolare, ha dovuto imparare a fare opposizione in Parlamento, soprattutto nei confronti diretti con un leader della statura di Margaret Thatcher.

Prima del 1989, infatti, l'opposizione parlamentare laburista era abbastanza indifferente alle proprie performances in Parla- 
mento, perché condizionata da lunghi anni di assenteismo, senso di frustrazione, passività (Kavanagh 1989). Ma proprio in quell'anno il governo ombra si è adeguato e rinnovato, con velocità impressionante, alla nuova situazione e ai nuovi compiti derivanti dagli obiettivi politici generali di rinnovamento del partito. Si è così assistito negli ultimi due anni ad un intenso turnover nel governo-ombra, che ha portato in primo piano, a posizioni di responsabilità, personaggi di grande competenza universalmente riconosciuta (come lo scozzese John Smith al Ministero dello Scacchiere), e giovani di notevole capacità e dinamismo. Per la prima volta poi, nel 1989, è stata assegnata una quota minima di tre posti all'interno del governo-ombra alle donne, superando la tradizionale chiusura verso la dimensione della rappresentanza di genere in una pur sempre austera istituzione.

Il nuovo gruppo dirigente ha innovato, inoltre, nel confronto con il governo, il ventaglio dei temi di intervento e di critica, immettendo nuovi temi come l'ambiente, l'equità sociale, le opportunità per le donne e le famiglie, i diritti dei consumatori nei confronti dei pubblici servizi, la dimensione sociale della partecipazione alla Comunità europea, accanto alla tradizionale difesa delle conquiste del Welfare State (del National Health Service anzitutto, ad un certo punto in pericolo di essere riformato in chiave privatistica). L'efficacia di questo nuovo «stile» e modo di fare opposizione in Parlamento da parte dei frontbenchers è stata generalmente riconosciuta dalla stampa e dai commentatori politici, contribuendo ai successi del partito in questi anni. Da notare, inoltre, che i personaggi di spicco del governo-ombra, diversamente dal passato quando il PLP era autonomo ma separato dal resto delle strutture di partito, sono stati anche $i$ protagonisti, pienamente coinvolti e responsabili, di tutto il processo della Policy Review. L'essere dentro la struttura decisionale del partito extraparlamentare ha enormemente rafforzato il prestigio e l'autorevolezza del governo-ombra, non più visto come una struttura separata e lontana dalla base e dagli apparati.

Il rilancio della centralità del Parliamentary Labour Party e delle sue strutture è avvenuto anche sul piano dei poteri formali o statutari, con ciò recuperando rapidamente il terreno perduto con le riforme organizzative del 1979-81. Al governo-ombra, ad esempio, è stato attribuito il potere di emendare il pacchetto di proposte inserite nella Policy Review e dibattute nel corso delle 
Conferences annuali, mentre queste ultime - pur ritenute il parlamento del partito, cioè organo sovrano - non hanno lo stesso potere. Il principio ispiratore di questa misura è che il partito nella sua funzione di governo, che si esplica anche dall'opposizione, non può essere vincolato da corpi esterni a quelli parlamentari. Viene qui riaffermata la teoria classica del responsible party government, come governo responsabile innanzitutto verso l'elettorato e verso il parlamento e non verso le organizzazioni extraparlamentari di partito che, come tali, sono considerate associazioni private cui non può essere riconosciuto un potere «mandatario» (Birch 1965).

La leadership, infatti, si è sempre rifiutata di sentirsi vincolata o condizionata - nell'eventualità di un governo laburista dalle decisioni della Conference di partito, in nome della responsabilità del governo anzitutto verso il paese, l'elettorato e il Parlamento. Quando - per citare il caso più recente - la Conference di Brighton (29 settembre-4 ottobre 1991) ha approvato a maggioranza una mozione che impegnava il partito a tagliare le spese della difesa in linea con la media europea, Kinnock ha ribadito che un eventuale governo laburista avrebbe deciso autonomamente e discrezionalmente su tale questione, in relazione a tutta una serie di valutazioni di stretta competenza governativa («The Times», 30 ottobre 1991).

La riconquista di poteri del PLP e la rivendicazione di autonomia della leadership indicano, assieme a problemi più generali nel rapporto tra democrazia rappresentativa e partiti politici, che i problemi del rinnovamento di un partito socialista sono anche, nella misura in cui si pone come alternativa di governo, problemi di strategia istituzionale e di funzionalizzazione a questa dei meccanismi decisionali interni. Anche se l'equilibrio dei poteri e il modello di democrazia interni al Labour non sono più quelli descritti da McKenzie - giacché il modello organizzativo tende a trasformarsi verso un partito di massa a base di iscrizioni individuali, e comunque le riforme organizzative del 1979-81 hanno portato a profonde modificazioni - tuttavia è significativo come nell'azione complessiva di rinnovamento il partito abbia dovuto riadattarsi alla logica di funzionamento del «modello Westminster». 
La riforma organizzativa: un nuovo modello di partito

La riforma del vecchio e tradizionale modello del partito laburista - un punto anche questo trattato a parte dalla Policy Review - si è indirizzata ad obiettivi di modernizzazione e di democratizzazione. Modernizzazione perché l'intera macchina organizzativa appare arcaica, legata ancora allo statuto del 1918 , fossilizzata e appesantita nelle sue procedure interne. Democratizzazione perché il partito, come struttura federata, non è una struttura democratica in quanto le decisioni sono prese fondamentalmente dai sindacati il cui block vote ammonta a circa il $90 \%$ dei voti raccolti nella Conference. Anche a livello dei collegi elettorali il peso del block vote delle unions nella selezione dei parlamentari risulta preponderante. Questo carattere non democratico del Labour è stato ampiamente riconosciuto nella letteratura scientifica sul partito e dalla stessa dirigenza (Massari 1989 e 1991).

Questi due obiettivi generali sono stati così presentati e articolati nella Conference di Brighton del 1989:

- necessità di un passaggio graduale da un partito federato a un partito di massa a base individuale, in cui sia possibile praticare una diretta partecipazione democratica degli iscritti al processo decisionale. La democrazia interna basata sulla partecipazione degli iscritti è anche un modo di rompere l'influenza negativa e il predominio degli attivisti a livello locale e nei vari processi decisionali. Un partito di massa, di stampo europeo, è anche un modo di avvicinare il partito all'elettorato, nel presupposto che quanto più larga è la rappresentanza sociale degli iscritti tanto più essa riflette lo spettro dell'intera popolazione;

- lancio di una campagna di reclutamento di massa di membri individuali che nel 1991 avrebbero dovuto raggiungere la cifra di un milione di iscritti. Contestualmente, già nel 1988, si era cambiato il sistema di reclutamento: dai singoli collegi questo veniva affidato direttamente agli uffici centrali del partito, che lo avrebbero gestito tramite una propaganda centrale (per posta, con pubblicità sui giornali nazionali ecc.) e una computerizzazione unificata (in modo da avere anche dati informativi di varia natura dei propri iscritti). Bisogna dire, però, che alla data della Conference del settembre 1991, questo obiettivo è largamente fallito. Gli iscritti individuali sono stati, infatti, solo 311.152 (1988: 265.927) e il sistema computerizza- 
to si è spesso inceppato (NEC Report 1991, London, Labour Party);

- riduzione, contestualmente alla crescita del partito di massa a base individuale, del potere decisionale dei sindacati, abbassando gradualmente la quota del block vote. A livello locale questa quota è stata già portata al $40 \%$, mentre ai constituency parties spetta il $60 \%$. Nella Conference di Brighton si è approvata una mozione tesa al restringimento del block vote per il livello nazionale fino al $50 \%$. Ma in realtà esistono varie ipotesi di soglie e niente ancora di definitivo è stato deciso (NEC Report 1989);

- cambiamento delle procedure di elezione dei membri spettanti ai constituency parties all'interno del NEC ( 7 su 31 complessivi e di cui 12 eletti dalle unions). Questi non devono più essere designati dai comitati locali di partito (quindi sostanzialmente solo da funzionari e attivisti), ma designati dalle organizzazioni locali attraverso ballots (ossia votazioni a scrutinio segreto) tra gli iscritti. In genere i ballots favoriscono candidati più moderati e penalizzano quelli più estremisti o percepiti come tali;

- riforma delle regole, procedure, sistema di voto della Conference. Questa, più che un vero congresso di partito secondo lo stile dei partiti europei, è una sorta di sessione parlamentare del partito, con tutti i vizi del «parlamentarismo». Si tiene annualmente, non esiste una agenda precisa dei suoi lavori; le sue procedure e regole sono contorte, macchinose, contraddittorie, spesso poco conosciute dai partecipanti. Il suo ruolo decisionale, inoltre, non è affatto chiaro, in quanto risultante sia dallo statuto del 1918 - secondo il quale la Conference è l'istanza decisionale sovrana del partito - sia da una sedimentazione di convenzioni e pratiche in tutt'altra direzione. Il conflitto sull'attribuizione del maggior ruolo decisionale alla Conference o al Parliamentary Party è una costante della storia del Labour, e non si è mai arrivati ad una conclusione definitiva. Questa riforma, comunque, guarda al modello europeo di congresso di partito. E una delle prime misure dovrebbe essere quella di tenerlo non più a scadenza annuale;

- infine, cambiamenti sono previsti sul terreno della rappresentanza, stabilendo quote per le donne nei vari organismi (ma ancora, a parte lo Shadow Cabinet, nella maggior parte di questi non è stata fissata alcuna quota vincolante, tanto che nella elezione del NEC nella Conference del 1991 nessuna donna è entrata a farne parte, suscitando nel partito scandalo e polemi- 
che) e permettendo la costituzione di associazioni affiliate composte solo da membri di colore (nel passato tale richiesta, proveniente dalle stesse minoranze di colore, era sempre stata respinta in nome dell'integrazione e contro il separatismo).

C'è da osservare, conclusivamente, che tra tutte le dimensioni dell'innovazione quella dell'organizzazione si è rivelata la più difficile da trattare. Il limite più macroscopico, infatti, è costituito dalla grande difficoltà ad operare la trasformazione da un partito federato ad un partito di massa a base individuale. $\mathrm{Ci}$ sono ovviamente limiti oggettivi, ma anche una enorme resistenza di molte componenti a riformarsi o a cedere quote di potere (come i sindacati) (Taylor 1989). Soprattutto viene in campo la forza d'inerzia dell'organizzazione e di mentalità sedimentatesi in molti decenni (è il caso della riforma della Conference e di quella relativa alle quote femminili).

\section{Conclusioni}

Una valutazione d'assieme indica che il processo di rinnovamento di un partito politico - nella fattispecie un partito di sinistra, socialista, a struttura indiretta e di opposizione - è un processo complesso e difficile, ma non impossibile. Esso richiede l'apporto sì della leadership, ma l'impulso può venire anche da altri soggetti (esperti delle comunicazioni, sindacati ecc.). Esso, se vuole essere profondo e duraturo, ha bisogno di tempo, deve essere necessariamente graduale per garantire l'unità e il consenso interni. Il rinnovamento ha, poi, bisogno di competenze, di conoscenze, di dati empirici, di analisi quantitative e qualitative. Il partito deve confrontarsi con i trend sociali, ma dotarsi anche di una strategia istituzionale congruente e coerente con il contesto della competizione politica e della struttura istituzionale del paese. Un partito è composto di iscritti, militanti, strutture, elettorato ecc., ha molteplici funzioni (di rappresentanza, di decisione, di socializzazione ecc.), tutto questo richiede non un'unica strategia di intervento, ma una strategia coordinata a più dimensioni.

Se la valutazione del rinnovamento del partito laburista secondo le linee descritte - va rapportata agli effetti sul e nel sistema politico, il giudizio può risultare più obiettivo. Il rinnovamento del partito - che ha indubbiamente implicato, al di là di giudizi di valore, uno «spostamento al centro» - ha condiziona- 
to tutta la dinamica del sistema politico britannico negli ultimi anni. Intanto, ha evitato la minaccia del «riallineamento» a favore dei partiti di centro, proprio perché ha rioccupato spazi prima lasciati scoperti dall'eccessivo spostamento a sinistra e dalla radicalizzazione delle politiche. Sta di fatto che dal 1988 in poi il sistema politico è tornato a funzionare come il tradizionale two-party system (Are e Ceccanti 1992), e - dopo il cambio di leadership dalla Thatcher a Major - anche come un sistema fondato sulla consensus politics, giacché la distanza ideologica tra i due maggiori partiti si è notevolmente ridotta. Rimane certamente una aspra competizione tra questi, dove i rispettivi valori sono confrontati e non eliminati, ma ciò fa parte del gioco democratico e delle regole del bipartitismo britannico.

In tutti i tipi di elezioni tenutesi dal 1987 al 1991 il partito laburista ha guadagnato consensi, confermando il suo forte recupero come effetto del suo rinnovamento (by-elections, elezioni europee del 1989, in cui è divenuto il primo partito, elezioni amministrative del 1990 e 1991, in cui i conservatori hanno subito notevoli perdite e $\mathrm{i}$ laburisti significativi progressi).

\section{Poscritto}

Dopo che questo saggio era già stato completato, il 9 aprile 1992 si sono tenute in Gran Bretagna le elezioni politiche generali. I risultati sono stati i seguenti: i conservatori con il $43 \%$ dei voti ottengono 336 seggi e una maggioranza di governo di 21 seggi; i laburisti il $35 \%$ dei voti e 271 seggi; i liberaldemocratici il $18 \%$ dei voti e 20 seggi. Nonostante, dunque, l'enorme sforzo di innovazione politica condotto a partire dal 1983, il partito laburista manca clamorosamente e inaspettatamente il test decisivo per l'opposizione nel sistema politico britannico: la capacità di divenire maggioranza di governo. All'indomani dei risultati elettorali Neil Kinnock ha annunciato le sue dimissioni da leader del partito e il 18 luglio 1992 lo scozzese John Smith viene eletto alla leadership.

I risultati di queste elezioni sono stati, senza dubbio, sorprendenti e ricchi di innumerevoli implicazioni non solo per il Labour, ma anche per la politica britannica, la sua forma di governo (si parla sempre più di fine del modello dell'alternanza e di one-party-State) e per la stessa politica europea (la 
Gran Bretagna ha oggi il più stabile governo di tutta l'Europa occidentale). L'esito di queste elezioni infatti, per tutta la durata della campagna elettorale, non era stato previsto da nessuno.

Tutti i responsi dei circa sessanta sondaggi tenuti durante la campagna avevano, nella stragrande maggioranza, indicato o una vittoria dei laburisti o un risultato che avrebbe portato ad un bung parliament (un parlamento, cioè, in cui nessun partito riesce ad ottenere la maggioranza assoluta dei seggi). Ma accanto ai sondaggi, tutti gli osservatori, gli opinionisti, i mass-media convergevano nella previsione di una sconfitta dei conservatori. Si pensi che persino dopo la chiusura dei seggi elettorali alle 10 di sera di giovedì 9 aprile, le cosiddette exit polls (10.000 elettori intervistati all'uscita dei seggi) davano una previsione di un bung parliament. Dalle elezioni non è uscito sconfitto solo il Labour ma anche il mondo dei cosiddetti esperti. Ogni previsione, condotta con gli strumenti tecnici e scientifici dei sondaggi di opinione, è stata severamente smentita dai fatti, come mai era avvenuto prima in Gran Bretagna. Questa circostanza - meritevole in altra sede di un approfondimento specifico - segnala come in determinati momenti la politica sia qualcosa di estremamente più complesso e forse più misterioso di quanto non pretendano le rilevazioni scientifiche dei sondaggi di opinione.

Mentre per le sconfitte del partito laburista del 1979, 1983 e 1987 si possono trovare spiegazioni facilmente comprensibili ed esaustive, non è così per quella del 1992. In questo caso tutte le circostanze erano favorevoli all'opposizione e sfavorevoli al partito al governo. Elenchiamole sinteticamente: a) la presenza di una recessione economica durissima e senza precedenti sin dagli anni ' 30 , con quasi 3 milioni di disoccupati, con la chiusura nell'ultimo anno di circa 50.000 aziende, con tassi di interesse altissimi sui mutui fondiari ecc.; b) diffuso risentimento nel paese verso i conservatori a causa della famigerata e odiata polltax; c) una elezione combattuta largamente sui temi propri del partito laburista, come il futuro del National Health Service e dei servizi pubblici dello Stato sociale; d) una campagna elettorale gestita pessimamente dai conservatori a fronte di quella dei laburisti, giudicata la migliore che questi abbiano fatto dal 1964; e) un Labour Party profondamente e radicalmente innovato nelle sue politiche e nella sua immagine, percepito nuova- 
mente come alternativa credibile di governo, spostatosi verso il centro dello schieramento politico, tanto da essere accusato di eccessivo moderatismo.

Le domande allora che sorgono spontanee, e che si sono chiesti tutti i commentatori, sono: perché, nonostante tutte queste condizioni favorevoli, il Labour ha perso? Quali le cause della sconfitta? E ancora, se il Labour non ha vinto ora, quando mai potrà vincere? E quali saranno le conseguenze sul sistema politico e sulla forma di governo dell'incapacità del Labour a produrre alternanza (al termine della legislatura iniziata nell'aprile 1992 i conservatori saranno stati ininterrottamente al governo per 18 anni consecutivi)?

Elettoralmente, l'obiettivo di strappare la vittoria ai conservatori non era certamente facile per i laburisti. Nelle elezioni del 1987 la distanza tra i primi e i secondi era stata di undici punti percentuali e la differenza in seggi di quasi 140 . In queste elezioni certamente la distanza si è accorciata, con i conservatori che perdono complessivamente 34 seggi e i laburisti che ne guadanano 39. Ora la differenza in seggi è ridotta a 65 (più che dimezzata), e la differenza in percentuale di voti si è ridotta a 7 punti. Complessivamente, mentre i conservatori mantengono la stessa percentuale del 1987, il Labour avanza di circa 4 punti. In un diverso contesto di sistema elettorale, ad esempio in un contesto proporzionale (la DC in Italia, primo partito nelle elezioni del 5-6 aprile 1992, è sotto il 30\%), il risultato del Labour non sarebbe stato per niente male e avrebbe largamente premiato la sua opera di rinnovamento. Ma nel contesto uninomi: nale del plurality system («first past the post»), la sconfitta prima ancora che numerica è politica. La sconfitta deriva dal mancato raggiungimento dell'alternanza. Le cause che possono avere determinato la sconfitta del partito laburista sono ancora poco chiare o forse fin troppo evidenti. C'è, infatti, chi sostiene che il Labour ha perso perché... Labour (ad esempio «The Times»), perché cioè un partito socialista con politiche di redistribuzione del reddito orientate alla giustizia sociale e legate al vecchio modo di pensare della tradizione classica socialdemocraticokeynesiana. Di fronte all'alternativa tra una visione solidaristica mitigata dalle esigenze del mercato (Labour) e quella individualistica corretta da considerazioni sociali (il conservatorismo di Major, diverso dal puro thatcherismo), gli elettori hanno scelto quest'ultima. In questo senso, sarebbe uscito sconfitto il sociali- 
smo, sia pure democratico. Se consideriamo poi che tutte le tornate elettorali svoltesi in Europa dopo il 1989 - dopo cioè il crollo del socialismo reale, simboleggiato dal Muro di Berlino hanno visto la sconfitta o l'arretramento dei partiti di ispirazione socialista, sia al governo che all'opposizione, - e questo si è verificato in Svezia, Germania, Francia, Belgio, Italia - c'è da chiedersi se non siamo entrati in un'epoca post-socialista (come molti osservatori già sostengono), se l'89 e i crollo del comunismo non abbia colpito a morte solo i partiti comunisti, ma abbia anche compromesso gravemente l'idea stessa del socialismo, pure se qualificato come democratico ed occidentale. In questo senso, la sconfitta del partito laburista andrebbe inserita in un ciclo politico internazionale che, dopo il terremoto geo-politico del 1989, vede la sinistra europea in ritirata e in difficoltà e che sta determinando ovunque cambiamenti profondi negli assetti dei sistemi partitici tradizionali in una misura e con una velocità come mai era accaduto dopo il secondo dopoguerra (Massari 1992b).

Sul piano, invece, delle spiegazioni interne, ma intrecciate a quelle internazionali, si impongono, oltre quelle di politica economica, anche quelle d'ordine politico-istituzionale. Queste fanno riferimento da un lato alla paura di molti elettori indecisi fino all'ultimo dell'ingovernabilità e della prospettiva di un cambiamento del «modello Westminster»: un bung parliament avrebbe dato vita o a un governo minoritario e di breve durata o a un instabile governo di coalizione tra laburisti-liberaldemocratici e nazionalisti scozzesi (la cui possibilità sarebbe stata legata all'introduzione di una riforma elettorale in senso proporzionale e alla fine dell'Unione con il riconoscimento dell'indipendenza alla Scozia, entrambe richieste avanzate dagli eventuali partner di governo dei laburisti). Considerazioni di natura politico-istituzionale si riferiscono anche alla straordinaria resistenza, tenuta e flessibilità del partito conservatore, in grado di sacrificare un leader di statura mondiale come la signora Thatcher, primo ministro in carica e mai sconfitto alle elezioni, e di adeguare velocemente il suo programma economico e sociale a nuove esigenze.

Insomma, quali che siano le spiegazioni della sconfitta laburista, una cosa emerge chiaramente, e cioè che mentre tradizionalmente era vera la massima secondo cui non è l'opposizione a vincere le elezioni ma il governo a perderle, ora sarebbe vero il contrario, ossia che non è il governo a vincerle ma l'opposizione a perderle. Il dato più eclatante è proprio questo: l'impossibilità dell'opposizione laburista a vincere le elezioni. 
Questo giudizio non toglie validità al riconoscimento della sua profonda innovazione seguita negli anni a partire dal 1983, ma impone di inserire tale riconoscimento in un ambito analitico in parte più complesso - le innovazioni hanno sempre effetti più lenti rispetto ai danni provocati da politiche sbagliate e alle immagini negative che si sono sedimentate nell'elettorato; in più l'innovazione perseguita dal Labour ha trovato un limite insormontabile nella natura della sua struttura organizzativa, non ancora un partito di massa a base individuale, ma anacronisticamente dipendente in larghissima misura dai sindacati. Si aggiunga infine il contesto storico prodotto dagli avvenimenti internazionali dell'Est europeo che forse segna l'ingresso, per ciò che attiene alla sinistra, in una fase storica post-socialista.

\section{Riferimenti bibliografici}

Allum, P. (1988), Il caso britannico, relazione presentata al convegno nazionale del Consiglio Italiano per le Scienze Sociali su Le trasformazioni dei partiti politici nelle società occidentali, Roma 26-27 settembre.

Are, G. e S. Ceccanti (1991), L'Inghilterra nell'ultimo decennio: le trasformazioni del bipartitismo, in «Il Politico», n. 4.

Bagehot, W. (1963-1967), The English Constitution, Glasgow, Collins.

Beer, S.H. (1965), Modern British Politics. A Study of Parties and Pressure Groups, London, Faber and Faber.

- (1982), Britain Against Itself. The Political Contradictions of Collettivism, London, Faber and Faber.

Berrington, H. (a cura di) (1984), Change in British Politics, London, Frank Cross.

Birch, A.H. (1965), Representative and Responsible Government. An Essay on the British Constitution, London, Allen and Unwin.

- (1985), The Theory and Practice of Modern British Democracy, in Jowell e Oliver (1985), pp. 77-102.

Blondel, J. (1967), Voters, Parties and Leaders, London, Penguin Books.

Bochel, J. e D. Denver (1983), Candidate Selection in the Labour Party. What the Selectors Seek, in «British Journal of Political Science», 13, pp. 45-69.

Bogdanor, V. (1983), Multy-Party Politics and the Constitution, Cambridge, Cambridge University Press.

Bradley, I. (1981), Breaking the Mould? The Birth and Prospects of the Social Democratic Party, London, Robertson. 
Butler, D. e D. Kavanagh (1984), The British General Election of 1983, London, Macmillan.

- (1988), The British General Election of 1987, London, Macmillan.

Coates, D. (1980), Labour in Power? A Study of the Labour Government 1974-79, London, Longman.

Cook, C. e I. Taylor (a cura di) (1980), The Labour Party. An Introduction to its History Structure and Politics, London, Longman.

Cox, G. (1987), The Efficient Secret: The Cabinet and the Development of Political Parties in Victorian England, Cambridge, Cambridge University Press.

Crewe, I. e B. Sarlvik (1983), The Decade of Dealignment, Cambridge, Cambridge University Press.

Crewe, I. e M. Harrop (1989), Political Communications: The General Election Campaign of 1987, Cambridge, Cambridge University Press.

Drucker, H.M. (1981), Changes in the Labour Party Leadership, in «Parliamentary Affairs», 4, pp. 369-391.

- (1983), Intra-party Democracy in Action: The Election of Leader and Deputy Leader by the Labour Party in 1983, in «Parliamentary Affairs», 3, pp. 283-300.

Dunleavy, P. e C. Husbands (1985), British Democracy at the Crossroads, London, Allen \& Unwin.

Edgell, S. e V. Duke (1991), A Measure of Thatcherism. A Sociology of Britain, London, Harper Collins Academic.

Finer, S.E. (a cura di) (1975), Adversary Politics and Electoral Reform, Oxford, Oxford University Press.

Franklin, M. (1985), The Decline of Class Voting, Oxford, Oxford University Press.

Geckie, J. e R. Levy (1988), Reselection, Activism and the Labour Party, in «The Political Quarterly», 4, pp. 477-485.

Guttsman, W.L. (1963), The British Political Elite, London, MacGibbon \& Kee.

Hall, S. (1985), Authoritarian Populism: A Reply to Jessop et al., in «New Left Review», 151, pp. 115-124.

Hamilton, M.B. (1989), Democratic Socialism in Britain and Sweden, London, Macmillan.

Hanson, C.G. (1991), Taming the Trade Unions, London, Macmillan.

Harrop, M. (1988), Voting and the Electorate, in H. Drucker et al. (a cura di), Developments in British Politics 2, London, Macmillan, pp. 34-58.

Heath, A., R. Jowell e J. Curtice (1985), How Britain Votes, London, Pergamon Press.

Hennessy, P. e A. Seldon (a cura di) (1987), Ruling Performance. British Governments from Attlee to Thatcher, Oxford, Basil Blackwell. 
Howell, D. (1976), British Social Democracy, London, Croom Helm.

Hughes, C. e P. Wintour (1990), Labour Rebuilt: The New Model Party, London, Fourth Estate.

Jenkins, P. (1989), Mrs Thatcher's Revolution. The End of the Socialist Era, London, Pan Books.

Jessop, B. et al. (1987), Thatcherism, London, Polity Press.

Johnson, N. (a cura di) (1977), In Search of the Constitution: Reflections on State and Society in Britain, Oxford, Pergamon Press.

Jowell, J. e D. Oliver (a cura di) (1985), The Changing Constitution, Oxford, Clarendon Press.

Kavanagh, D. (a cura di) (1982), The Politics of the Labour Party, London, Allen \& Unwin.

- (1989), The Changing Political Opposition, in D. Kavanagh e A. Seldon (a cura di), The Thatcher Effect, Oxford, Clarendon Press, pp. 89-100.

- (1990), Thatcherism and British Politics, Oxford, Oxford University Press.

Kavanagh, D. e P. Morris (1989), Consensus Politics from Attlee to Thatcher, Oxford, Basil Blackwell.

Kogan, D. e M. Kogan (1983), The Battle for the Labour Party, London, Kogan Page.

Laver, M. (1989), Party Competition and Party System Change, in «Journal of Theoretical Politics», 3, pp. 301-324.

Mair, P. (1983), Adaptation and Control: Towards an Understanding of Party and Party System Change, in H. Daalder e P. Mair (a cura di), Western European Party Systems. Continuity and Change, London, Sage, pp. 405-430.

Massari, O. (1989), Il Labour Party dalla politica del «ghetto» al rinnovamento, in AA.VV, Sinistra Europea. Annali 1988-1989, Milano, Angeli, pp. 181-219.

- (1990a), Natura e ruolo delle opposizioni politico-parlamentari, in G. Pasquino (a cura di), Opposizione governo-ombra alternanza, Roma-Bari, Laterza, pp. 27-88.

- (1990b), La Gran Bretagna e la Comunità Europea: un'altra visione dell'Europa?, Working Papers dell'ISPI, Milano.

- (1991), Da partito di opposizione a partito di governo: il rinnovamento del Labour Party nel 1989, in AA.VV., Politica Europea. Annali 1990-1991, Milano, Angeli, pp. 161-188.

- (1992a), I partiti politici in Gran Bretagna tra organizzazione interna e «modello Westminster», in «Quaderni Costituzionali», 1, pp. 107-124.

- (1992b), Il voto in Europa: cosa sta succedendo?, in «Appunti di cultura e di politica», 5, maggio, pp. 26-32.

McKenzie, R.T. (1963), British Political Parties, London, Mercury Books. 
- (1982), Power in the Labour Party: The Issue of «Intra-Party Democracy», in Kavanagh (1992), pp. 202-222.

McLean, I. (1988), Ships that Pass in the Night: Electoral Reform and Social Choice Theory, in «The Political Quarterly», 1, pp. 63-71.

Mitchell, A. (1983), Can Labour Come in from the Cold?, in «Contemporary Review», 1414, pp. 240-245.

- (1989), Televising of The House of Commons, in «The House Magazine's Viewers's Guide», 20 novembre.

Norton, P. (1982), The Constitution in Flux, Oxford, Basil Blackwell.

Panebianco, A. (1982), Modelli di partito, Bologna, il Mulino.

Riddel, P. (1989), Cabinet and Parliament, in D. Kavanagh e A. Seldon (a cura di), The Thatcher Effect, Oxford, Clarendon Press, pp. 101-113.

Robertson, D. (1984), Class and the British Electorate, Oxford, Basil Blackwell.

Seyd, P. (1990), Party Renewal: The British Labour Party, relazione presentata al Workshop su The European Socialist Parties, Università di Barcellona, ottobre.

Shaw, E. (1988), Discipline and Discord in the Labour Party. The Politics of Managerial Control in the Labour Party 1951-87, Manchester, Manchester University Press.

- (1989), The Policy Review and Labour's Policy-making system, relazione presentata alla Conferenza annuale della Political Studies Association, University of Warwick, 4-6 aprile.

Smith, M.J. (1991), A Return to Revisionism? The Labour Party's Policy Review, relazione presentata alla Conferenza annuale della Political Studies Association, University of Lancaster, 15-17 aprile. 